\title{
ON SOME THEOREMS OF LITTLEWOOD AND SELBERG II
}

\author{
K. Ramachandra and A. Sankaranarayanan
}

\section{Introduction}

In a previous paper with the same title [1] we proved some theorems about the Riemann zeta-function under the assumption of Riemann hypothesis. In this paper we prove some unconditional results on $\zeta(s)$. Stating somewhat more generally we prove the following.

Theorem. Let $s=\sigma+i t$ and

$$
F(s)=\sum_{n=1}^{\infty} \frac{a_{n}}{n^{s}}=\prod_{p}\left(1-\frac{\omega(p)}{p^{s}}\right)^{-1} \quad(\sigma>1),
$$

where $p$ runs through all primes and $\omega(p)$ are some complex numbers (independent of $s$ ) with absolute value not exceeding 1 . Suppose $\alpha$ and $\delta$ are positive constants satisfying $\frac{1}{2} \leq \alpha \leq 1-\delta$ and that in $\{\sigma \geq \alpha-\delta, T-H \leq t \leq T+H\} F(s)$ can be continued analytically and there $|F(s)|<T^{A}$. Here $A$ is a positive constant, $T \geq T_{0}, H=C \log \log \log T$ where $T_{0}$ and $C$ are large positive constants. Let $F(s) \neq 0$ in $\{\sigma>\alpha, T-H \leq t \leq T+H\}$. Then for $\alpha+\delta \leq \sigma \leq 1-\delta, t=T$, we have

$$
\frac{F^{\prime}(s)}{F(s)}=O\left((\log T)^{\Theta}\right)
$$

and

$$
\log F(s)=O\left((\log T)^{\Theta}(\log \log T)^{-1}\right),
$$

where $\Theta=(1-\sigma) /(1-\alpha)$.

Remark 1. The application to $\zeta(s)$ is immediate by density results. By standard methods we can also prove density results for $F(s)$ provided in, say $\left\{\sigma \geq 3 / 4, t \geq T_{0}\right\} F(s)$ can be continued analytically and there $|F(s)|<t^{A}$.

Remark 2. The theorem can be generalised further by allowing some growth condition for $\omega(p)$. We can state our theorem in a slightly different way to allow $F(s)=L(s, \chi)$ for characters $\chi(\bmod q)$, for example for $|t| \leq q$. 
Remark 3. We can state a result for $\alpha+\delta \leq \sigma \leq 1+\delta$ analogous to the remark made by D.R. Heath-Brown in Section 14.33 of [3].

Remark 4. In a later paper with the same title we hope to obtain inequalities dealing with $|\arg F(\sigma+i t)|$ for $\sigma \geq \alpha$ and $\log |F(\sigma+i t)|$ for $\sigma>\alpha$, analogous to what we proved in [1].

Remark 5. The $t$-interval condition $T-H \leq t \leq T+H$ is made possible by the kernel function $\exp \left((\sin w)^{2}\right)$ used extensively by Ramachandra in his papers.

\section{Notation}

In Lemmas 1 and 2 we borrow results from [4] and [3] in the same notation. But in Lemma 2 we have changed the result contained in [3] to suit our needs (see Remark below Lemma 2). We use $z=x+i y, w=u+i v$ and $s=\sigma+i t$ in various contexts and we hope this does not cause confusion. For any analytic function $F(s)$ we write $\left(F^{\prime} / F\right)(s)$ for $F^{\prime}(s) / F(s)$. The symbol $\equiv$ denotes a definition.

Lemma 1. Let $f(z)$ be analytic in $|z|<R$. Suppose $f(0)$ is different from zero. For $0 \leq x<R$ let $n(x)$ denote the number of zeros of $f(z)$ in $|z| \leq x$. Then for $0 \leq r<R$ we have

$$
\int_{0}^{r} n(x) \frac{d x}{x}=\frac{1}{2 \pi} \int_{0}^{2 \pi} \log \left|\frac{f\left(r e^{i \theta}\right)}{f(0)}\right| d \theta .
$$

Remark. This result is called Jensen's theorem. For its proof see pages 124 to 126 of [4].

Lemma 2. If $f(s)$ is regular and

$$
\left|\frac{f(s)}{f\left(s_{0}\right)}\right| \leq e^{M} \quad(M>1)
$$

in $\left|s-s_{0}\right| \leq r$, then for any constant $\epsilon$ (with $0<\epsilon<\frac{1}{2}$,) we have

$$
\left|\frac{f^{\prime}}{f}(s)-\sum_{\rho} \frac{1}{s-\rho}\right| \ll_{\epsilon} \frac{M}{r} \quad \text { in }\left|s-s_{0}\right| \leq(1-2 \epsilon) r
$$

where $\rho$ runs over all zeros of $f(s)$ such that

$$
\left|\rho-s_{0}\right| \leq(1-\epsilon) r
$$

Remark. From Lemma 1 above and the concluding remarks in [2] on (24), (25) and (26) of that paper we obtain Lemma 2 which is nearly contained as Lemma 2 of Section 3.9 of [3]. 
Lemma 3. Let $z=x+i y$ be a complex variable and

$$
F(z)=\sum_{n=1}^{\infty} \frac{a_{n}}{n^{z}}=\prod_{p}\left(1-\frac{\omega(p)}{p^{z}}\right)^{-1} \quad(x>1),
$$

where $p$ runs over all primes and $\omega(p)$ are complex numbers independent of $z$ with $|w(p)| \leq 1$. Let $F(z)$ be regular in $\{x \geq \alpha-\delta, T-H \leq y \leq T+H\}$ and there $|F(z)|<T^{A}$. Here $T \geq T_{0}, \frac{1}{2} \leq \alpha \leq 1-\delta, H=C \log \log \log T$ where $\delta$ is a small positive constant, $\alpha$ is a positive constant, and $T_{0}$ and $C$ are large positive constants. Put $z_{0}=2+i y_{0}$ where $T-\frac{1}{2} H \leq y_{0} \leq T+\frac{1}{2} H$. Then for $z_{1}=x_{0}+i y_{0}$ with $\alpha-\delta_{1} \leq x_{0} \leq 2$, we have,

$$
\left|\frac{F^{\prime}}{F}\left(z_{1}\right)-\sum_{\rho \in D} \frac{1}{z_{1}-\rho}\right| \ll \log T,
$$

where $\rho$ runs over all the zeros of $F(z)$ in the disc $D=D\left(z_{0}, 2-\alpha+2 \delta_{1}\right)$ defined by

$$
\left|z-z_{0}\right| \leq 2-\alpha+2 \delta_{1}
$$

Here $\delta_{1}$ is any positive constant such that $2 \delta_{1}<\delta$. In particular the lemma holds for $z_{1}=\alpha+i y_{0}$.

Remark. The lemma is trivially true for $z_{1}=x_{0}+i y_{0}$ with $x_{0} \geq 1+\delta$.

\section{Proof of the theorem}

Lemma 4. Let $s=\sigma+i t$ where $\alpha+\delta_{1} \leq \sigma \leq 1-\delta_{1}, w=u+i v$, $2 \leq X \leq \exp (10(\log \log T) /(1-\alpha)), B \geq 10000$. Then

$$
\begin{gathered}
I \equiv \frac{1}{2 \pi i} \int_{u=2} \frac{F^{\prime}}{F}(s+w) X^{w} \exp \left(\left(\sin \frac{w}{B}\right)^{2}\right) \frac{d w}{w} \\
=O\left(X^{1-\sigma}\right) .
\end{gathered}
$$

Proof. The proof follows from

$$
\frac{1}{2 \pi i} \int_{u=2}\left(\frac{X}{n}\right)^{w} \exp \left(\left(\sin \frac{w}{B}\right)^{2}\right) \frac{d w}{w}=1+O\left(\frac{n}{X}\right) \text { or } O\left(\frac{X}{n}\right) .
$$

according as $n \leq X$ or $n>X$. 
Lemma 5. Let $3 V \sim H$ and $|v| \leq V^{\prime}$ ( $V^{\prime}$ will be chosen to be asymptotic to $V$ ). Then for (fixed $s=\sigma+i$ and all $w=u+i v$ ), $u+\sigma \geq \alpha-\delta_{1}$, we have,

$$
\left|\frac{F^{\prime}}{F}(s+w)-\sum_{\rho} \frac{1}{s+w-\rho}\right| \ll \log T
$$

where $\rho$ runs over all the zeros of $F(z)$ in the disc $D=D\left(z_{0}, 2-\alpha+2 \delta_{1}\right)$ defined by $\left|z-z_{0}\right| \leq 2-\alpha+2 \delta_{1}$ where $z_{0}=2+i t+i v$.

Proof. The proof follows from Lemma 3.

Lemma 6. Let

$$
\mu(\rho)=\frac{2^{s+w-\rho}-1}{(s+w-\rho)^{2} \log 2}
$$

and

$$
\mu=\sum_{\rho} \mu(\rho)
$$

where $\rho$ runs over all the zeros of $F(z)$ in the rectangle $R$ defined by

$$
R:\left\{\operatorname{Re} z \geq \alpha-2 \delta_{1},|t-y| \leq 2 V\right\} .
$$

Then for $|v| \leq V^{\prime}$ ( $V^{\prime}$ will be chosen asymptotic to $V$ ) and $u+\sigma \geq \alpha-\delta_{1}$ we have,

$$
\left|\frac{F^{\prime}}{F}(s+w)-\mu\right| \ll \log T .
$$

Proof. For $D$ as in Lemma 5, we have

$$
\sum_{\rho \in D} \frac{1}{s+w-\rho}-\sum_{\rho \in D} \mu(\rho)=O(\log T),
$$

since (by Jensen's theorem) there are $O(\log T)$ zeros involved and for any fixed $\rho$

$$
\left|\frac{1}{s+w-\rho}-\mu(\rho)\right| \ll 1
$$

since it is so on $|s+w-\rho|=10$. Again

since for $\rho \notin D$, we have

$$
\left|\sum_{\rho \notin D, \rho \in R} \mu(\rho)\right| \ll \log T
$$

$$
\begin{aligned}
|s+w-\rho| & \geq\left|z_{0}-\rho\right|-\left|s+w-z_{0}\right| \\
& \geq 2-\alpha+2 \delta_{1}-\left(2-\alpha+\delta_{1}\right)=\delta_{1}
\end{aligned}
$$

Lemma 7. It is possible to choose $V^{\prime} \sim V$ such that on $v= \pm V^{\prime}$ and $u+\sigma \geq \alpha-10 \delta_{1}$ we have,

$$
\left|\sum_{\rho \in R} \mu(\rho)\right| \ll(\log T)^{2}
$$

Here $10 \delta_{1}<\delta$ 
Proof. By Jensen's theorem the number of zeros of $F(z)$ in $\left\{x \geq \alpha-11 \delta_{1}, Y \leq\right.$ $y \leq Y+1\}$ with $11 \delta_{1}<\delta$ is $O(\log T)$ provided $T-2 V \leq Y \leq T+2 V$. Hence the number of zeros of $F(s+w)$ in $\left\{u+\sigma \geq \alpha-11 \delta_{1}, V-1 \leq v \leq V+1\right\}$ is $O(\log T)$ and so there exists a line $v=V^{\prime}$ such that on this line $|s+w-\rho| \gg 1 / \log T$. This proves Lemma 7 since the number of zeros in $2 \geq|s+w-\rho| \gg 1 / \log T$ is $O(\log T)$ and also the zeros $\rho$ with $|s+w-\rho| \geq 2$ contribute $O(\log T)$. The total contribution to $\mu$ is therefore $O\left((\log T)^{2}\right)$ and this proves Lemma 7 completely.

Lemma 8. We have (if there are no zeros of $F(z)$ in $x>\alpha$ and $T-H \leq$ $y \leq T+H)$

$$
\begin{aligned}
& I=\frac{F^{\prime}}{F}(s) \\
& +\frac{1}{2 \pi i} \int_{u=\alpha-\sigma}\left\{\left(\frac{F^{\prime}}{F}(s+w)-\mu\right)+\mu\right\} X^{w} \exp \left(\left(\sin \frac{w}{B}\right)^{2}\right) \frac{d w}{w}+o(1)
\end{aligned}
$$

where the integration is restricted to $|v| \leq V^{\prime}$ and we take the integral to mean the limit as we move from $u=2$ to $u=\alpha-\sigma$.

Proof. First, the contribution to $I$ of Lemma 4 from $|v| \geq V^{\prime}$ is $o(1)$. The lemma now follows on moving the line of integration to $u=\alpha-\sigma$ since by Lemmas 6 and 7 the horizontal bits contribute $o(1)$. Note that $\exp \left((\sin w)^{2}\right)$ decays like $(\exp \exp (|v| / 10))^{-1}$ uniformly in $|u| \leq 1 / 10$.

Lemma 9. We have,

$$
\int_{u=\alpha-\sigma,|v| \leq V^{\prime}}\left(\frac{F^{\prime}}{F}(s+w)-\mu\right) X^{w} \exp \left(\left(\sin \frac{w}{B}\right)^{2}\right) \frac{d w}{w}=O\left(X^{\alpha-\sigma} \log T\right) .
$$

Proof. The proof follows by Lemma 6 .

Lemma 10. We have

$$
J \equiv \frac{1}{2 \pi i} \int_{u=\alpha-\sigma,|v| \leq V^{\prime}} \mu X^{w} \exp \left(\left(\sin \frac{w}{B}\right)^{2}\right) \frac{d w}{w}=O\left(X^{\alpha-\sigma} \log T\right) .
$$

Proof. Let as before $11 \delta_{1}<\delta$. We move the line of integration to $u=$ $\alpha-\sigma-10 \delta_{1}$. We obtain

$$
\begin{aligned}
J= & \sum_{\rho \in R} \frac{X^{\rho-s}}{\rho-s} \exp \left(\left(\sin \frac{\rho-s}{B}\right)^{2}\right) \\
& +\frac{1}{2 \pi i} \int_{u=\alpha-\sigma-10 \delta_{1}} \mu X^{w} \exp \left(\left(\sin \frac{w}{B}\right)^{2}\right) \frac{d w}{w}
\end{aligned}
$$

the last integration being subject to $|v| \leq V^{\prime}$. Now since $\operatorname{Re} \rho \leq \alpha$ and $\sigma \geq \alpha+\delta$ and $\exp \left((\sin w)^{2}\right)$ tapers in $|u| \leq 1 / 10$ uniformly as fast as $(\exp \exp (|v| / 10))^{-1}$, the lemma follows. 
Lemma 11. We have, uniformly for $\{\alpha+\delta \leq \sigma \leq 1-\delta, t=T\}$

$$
\frac{F^{\prime}}{F}(s)=O\left((\log T)^{\Theta}\right)
$$

provided $F(z) \neq 0$ in $\{x>\alpha, T-H \leq y \leq T+H\}$. Here $\Theta$ is as stated in the theorem.

Proof. The proof follows from Lemmas 4, 8, 9 and 10 on choosing $X$ by $X^{1-\alpha}=\log T$.

Lemma 12. Subject to the conditions of Lemma 11,

$$
\log F(s)=O\left((\log T)^{\Theta}(\log \log T)^{-1}\right)
$$

Proof. The proof follows by integrating (22) with respect to $\sigma$ from $\sigma$ to $\sigma^{\prime} \equiv \frac{1}{2}(1+\sigma)$, since (as will be proved in the next lemma) $\log F\left(\sigma^{\prime}+i t\right)=$ $O\left((\log T)^{\Theta-\epsilon}\right)$, for some fixed $\epsilon>0$.

Lemma 13. We have, with $\sigma^{\prime}=\frac{1}{2}(1+\sigma)$,

$$
\log F\left(\sigma^{\prime}+i t\right)=O\left((\log T)^{\Theta-\epsilon}\right)
$$

for some fixed $\epsilon=\epsilon(\sigma)>0$.

Proof. By a simple application of the Borel-Carathéodory theorem we have $\log F(\sigma+\epsilon+i t)=O(\log T)$ for $\left\{\sigma \geq \alpha, T-\frac{1}{2} H \leq t \leq T+\frac{1}{2} H\right\}$. Put $s^{\prime}=\sigma^{\prime}+i t$. We proceed by considering (as in Lemma 4) the integral

$$
\frac{1}{2 \pi i} \int_{u=2} F\left(s^{\prime}+w\right) X^{w} \exp \left(\left(\sin \frac{w}{B}\right)^{2}\right) \frac{d w}{w}
$$

and moving the portion $|v| \leq H / 3$ of the line of integration to $u+\sigma^{\prime}=\alpha+\epsilon$ i.e. $u=\alpha-\sigma^{\prime}+\epsilon$. This leads to the Lemma. With Lemmas 11, 12 and 13 the theorem stated in the introduction is completely proved.

Remark. If $F(s) \neq 0$ in $\{\sigma>\alpha, T-C \leq t \leq T+C\}$ where $\frac{1}{2} \leq \alpha \leq 1-\delta$ and here $|F(s)|<T^{A},(T \geq 10)$, it follows by the proof of Theorem 14.2 of [3] that if $C=C\left(\delta, \epsilon, \sigma_{0}\right)$ then uniformly in $\alpha<\sigma_{0} \leq \sigma \leq 1$ and $t=T$, we have $F(s)=O\left((\log T)^{\Theta+\epsilon}\right)$, where the $O$-constant depends only on $A, \sigma_{0}, \delta$ and $\epsilon$. 


\section{References}

[1] Ramachandra, K., and A. Sankaranarayanan: On some theorems of Littlewood and Selberg I. - To appear.

[2] Ramachandra, K., and A. Sankaranarayanan: Notes on the Riemann zeta-function. - To appear.

[3] TitchmaRsh, E.C.: The theory of the Riemann zeta-function. - Second edition (revised by D.R. Heath-Brown), Oxford Science Publication, 1986.

[4] Titchmarsh, E.C.: The theory of functions. - Second edition, Oxford University Press, 1939.

K. Ramachandra

Tata Institute of Fundamental Research

School of Mathematics

Homi Bhabha Road

Bombay 400005

India

Received 6 June 1990

\author{
A. Sankaranarayanan \\ Tata Institute of Fundamental Research \\ School of Mathematics \\ Homi Bhabha Road \\ Bombay 400005 \\ India
}

